\title{
Revistas científicas de las universidades nacionales de la Región Cajamarca
}

\section{Scientific journals of the national universities of the Cajamarca Region}

DOI: $10.46932 / s f j d v 2 n 3-077$

Received in: May 1st, 2021

Accepted in: Jun 30th, 2021

\section{Carlos Rios-Campos}

Investigador RENACYT. Doctor en Gestión Universitaria. Maestro en Administración. Ingeniero de Sistemas. Docente de la Universidad Nacional Toribio Rodríguez de Mendoza de Amazonas. Bagua, Perú.

E-mail: carlos.rios@untrm.edu.pe

\section{Patricia Mercedes Tapia Macias}

Magíster en Ciencias Internacionales y Diplomacia. Abogada. Guayaquil, Ecuador.

E-mail: patty_tapia56@yahoo.com

\section{Oscar Efrén Anchundia Gómez}

Magister en Educación Universitaria e Investigación Educativa. Universidad de Guayaquil. Facultad de Filosofia Letras y Ciencias de la Educación - Carrera Educación Básica. Guayaquil, Ecuador.

E-mail: oanchundia@gmail.con

\section{Gina Elizabeth Mera Moya}

Docente de Lengua y Literatura de Básica Superior y Bachillerato. Máster Universitario en Formación Internacional Especializada del Profesorado. Magister en Diseño Curricular. Unidad Educativa Sarah Flor Jiménez. Guayaquil, Ecuador.

E-mail: ginamera79@gmail.com

\section{Shirley Tatiana Bustamante Vilchez}

MSc. en Ingeniería Ambiental y Seguridad Industrial. Estudiante de Doctorado en Ciencias Ambientales. Bióloga. Consultora independiente en temas ambientales. Sullana, Perú.

E-mail: biotabu@hotmail.com

\section{Jannyna Reto Gómez}

Magíster en Docencia Universitaria, Currículum e Investigación. Universidad Nacional de Frontera. Sullana, Perú.

E-mail: jreto@unf.edu.pe

\section{Jury Yesenia Aquino Trujillo}

Maestra en Ingeniería de Sistemas. Docente de la Universidad Católica Santo Toribio de Mogrovejo. Chiclayo, Perú. E-mail: jaquino@usat.edu.pe

\section{Pilar del Rosario Rios Campos}

Docente Principal. Maestra en Ciencias con Mención en Informática y Sistemas. Ingeniera de Sistemas. Universidad Nacional Pedro Ruiz Gallo. Lambayeque, Perú.

E-mail: prios@unprg.edu.pe 


\title{
RESUMEN
}

Las universidades nacionales peruanas, en especial del interior del país, están dirigiendo sus esfuerzos en difundir los nuevos conocimientos mediante sus revistas científicas indexadas. Se planteó el objetivo general, análizar las revistas científicas de las universidades nacionales de la Región Cajamarca. Los objetivos específicos, identificar el cumplimiento de las características de la metodología Latindex, por parte de las revistas científicas y formular recomendaciones en la gestión de las mismas. Se ha empleando la metodología de Latindex, con sus 38 características de calidad del Catálogo 2.0. Resultados, las tres revistas científicas cumplen con más de 30 características de la metodología de Latindex. Conclusiones, las revistas científicas de la UNC y UNJ han logrado su indexación en Latindex, mientras que de la UNACH no tiene indexación alguna, por lo que su visibilidad es mínima. Se recomienda minimizar los índices de endogamia editorial y autoría de las revistas científicas de las universidades nacionales de la Región Cajamarca. Además, se debe indicar las políticas de acceso y reuso, detección de plagio, uso de estadísticas y políticas de preservación digital.

Palabras clave: Revista científica; Latindex; universidad; Cajamarca; metodología.

\begin{abstract}
Peruvian national universities, especially in the interior of the country, are directing their efforts to disseminate new knowledge through their indexed scientific journals. The general objective was set, to analyze the scientific journals of the national universities of the Cajamarca Region. The specific objectives, to identify the fulfillment of the characteristics of the Latindex methodology, by the scientific journals and to formulate recommendations in their management. The Latindex methodology has been used, with its 38 quality characteristics of the Catalog 2.0. Results, the three scientific journals comply with more than 30 characteristics of the Latindex methodology. Conclusions, the scientific journals of the UNC and UNJ have achieved their indexation in Latindex, while the UNACH has no indexing whatsoever, so its visibility is minimal. It is recommended to minimize the rates of editorial inbreeding and authorship of the scientific journals of the national universities of the Cajamarca Region. In addition, access and reuse policies, plagiarism detection, use of statistics and digital preservation policies must be indicated.
\end{abstract}

Keywords: Scientific journals; Latindex; university; Cajamarca; methodology.

\section{INTRODUCCIÓN}

Ha llegado el momento de defender el derecho a la ciencia como nadie lo ha hecho hasta ahora. Es fundamental que más instituciones y personas se comprometan en esta discusión -igual que lo han hecho en el pasado con otros derechos fundamentales- y ayuden, en conjunto, a definir estándares y recomendaciones para los países de todas partes del mundo (UNESCO, 2020).

Existen numerosos ejemplos en la literatura científica de cómo enfoques de open data permiten que investigadores académicos e industriales puedan unir sus fuerzas para hacer nuevos descubrimientos científicos que podrían tener un enorme impacto en la salud humana, por ejemplo, mediante el descubrimiento de nuevos medicamentos o derivados de medicamentos previos (Aleixandre-Benavent, Ferrer y Peset, 2019).

Los repositorios institucionales, en cambio, reúnen la producción científica o académica resultado de la actividad docente e investigadora de los miembros de una o varias instituciones, almacenando, 
preservando, divulgando y dando acceso abierto a los recursos depositados en ellos (Gonzalez-Argote y Garcia-Rivero, 2020).

Las revistas académico-científicas son uno de los principales canales de comunicación y difusión de los resultados de investigación y de institucionalización social de la ciencia en la mayoría de los campos del conocimiento, pero no todas tienen el mismo prestigio y grado de influencia en la comunidad científica. Su reconocimiento depende en gran medida de su calidad y su visibilidad (Miguel, 2011).

La divulgación de la ciencia, en forma de artículos científicos, ha dejado de ser una opción y se ha transformado en una necesidad para los docentes universitarios (Barbón et al., 2019). Además, debemos reconocer que, este es el papel de la publicación científica, que brinda por su sistema de revisión por pares, una mínima garantía de la calidad en la producción académica (Carvalho, Lima y Coeli, 2020).

Es imperativo cambiar la percepción de los estudiantes frente a la investigación, lo que se consigue dejando en evidencia a partir del quehacer diario que la investigación constituye un elemento trascendental para el ejercicio profesional y no es un aspecto desligado de las funciones clínicas (Cruz et al., 2020).

Según los reportes de Scopus Análisis y Scimago Research Group en cuanto a la evolución de la producción científica en general por regiones del mundo, Europa, EE.UU. y Asia lideran el ranking en producción. Pero lo interesante es que, en la última década, Latinoamérica está en franco crecimiento en comparación con los antes mencionados (Hernández y Navia, 2019).

Como consecuencia de la escasez de clasificaciones que sirvan de insumo a la evaluación, más allá de los tradicionales WoS y Scopus, se hace necesario proponer nuevos modelos con miradas diferentes de los contextos específicos de cada país, que tengan en cuenta la importancia que merecen las fuentes regionales y ofrezcan un peso importante en la evaluación, la visibilidad e impacto, por los rigurosos criterios que estas aplican en la selección de revistas (ejemplo Redalyc, SciELO y Latindex) (Gregorio-Chaviano, 2018).

El sistema de Información sobre las revistas de investigación científica, técnico-profesionales y de divulgación científica y cultural; LATINDEX o Sistema Regional de Información en Línea para Revistas Científicas de América Latina, el Caribe, España y Portugal se editan en estos países y áreas cuenta con dos servicios fundamentales el directorio que ofrece información y datos bibliográficos de contacto de todas las revistas registradas y el CATÁLOGO, compuesto por las revistas con más altos estándares de calidad de acuerdo con la metodología de Latindex (Rodríguez, Socorro y León, 2019).

Recientemente en el Perú, la investigación se ubica en lo más alto de la estructura jerárquica universitaria. La conducción académica camina parejo a la gestión de la investigación en la universidad, dado que no debe insistir más en la tendencia academizante y profesionalizante (Venegas, Esquivel y Turpo-Gebera, 2019). 
En los últimos 20 años la creación de las universidades peruanas ha tenido un crecimiento acelerado, así como los programas de posgrado, los cuales se han desarrollado en condiciones cuestionables, muy distantes a las políticas de calidad de otros países de la región. De 57 universidades en 1996 a 143 universidades en 2017, donde el número de universidades privadas casi duplica al número de universidades públicas (Casimiro, Casimiro y Casimiro, 2020).

El reporte y liderazgo de mujeres en publicaciones científicas peruana es aún minoritario y con un estancamiento en el tiempo, por lo que deben crearse redes de colaboración y capacitación que sean más inclusivas con las mujeres, para apoyar y dar sostenibilidad a este crecimiento (Centeno-Leguía, MoralesConcha, Lopez y Mejia, 2020).

En Cusco, Perú, la producción científica de docentes universitarios de la Escuela de Medicina de la UNSAAC es baja. Ser coordinador de algún curso incrementó las posibilidades para publicar en SciELO y LIPECS (Chachaima-Mar, Fernández-Guzmán y Atamari-Anahui, 2019).

Las tres universidades nacionales de la Región Cajamarca son las siguientes: La Universidad Nacional Autónoma de Chota (UNACH), fue creada mediante Ley $\mathrm{N}^{\circ} 29531$, publicada con fecha 11 de mayo de 2010, es persona jurídica de derecho público sin fines de lucro, con autonomía académica, económica, normativa y administrativa con forme a Ley..., actualmente están en funcionamiento sus dos Campus Universitarios en Colpa Matara y Colpa Huacaris, ambos con su respectivo Plan Maestro de extensión e infraestructura, estamos regidos por la constitución política del Perú, por la Ley Universitaria $\mathrm{N}^{\circ} 30220$, por la Ley de su creación $\mathrm{N}^{\circ} 29531$ y por la Resolución $\mathrm{N}^{\circ}$ 160-SUNEDU-2018/CD de fecha 8 de diciembre de 2018 que le otorga el licenciamiento por un lapso de seis (6) años desde la publicación de la misma (UNACH, 2018). 

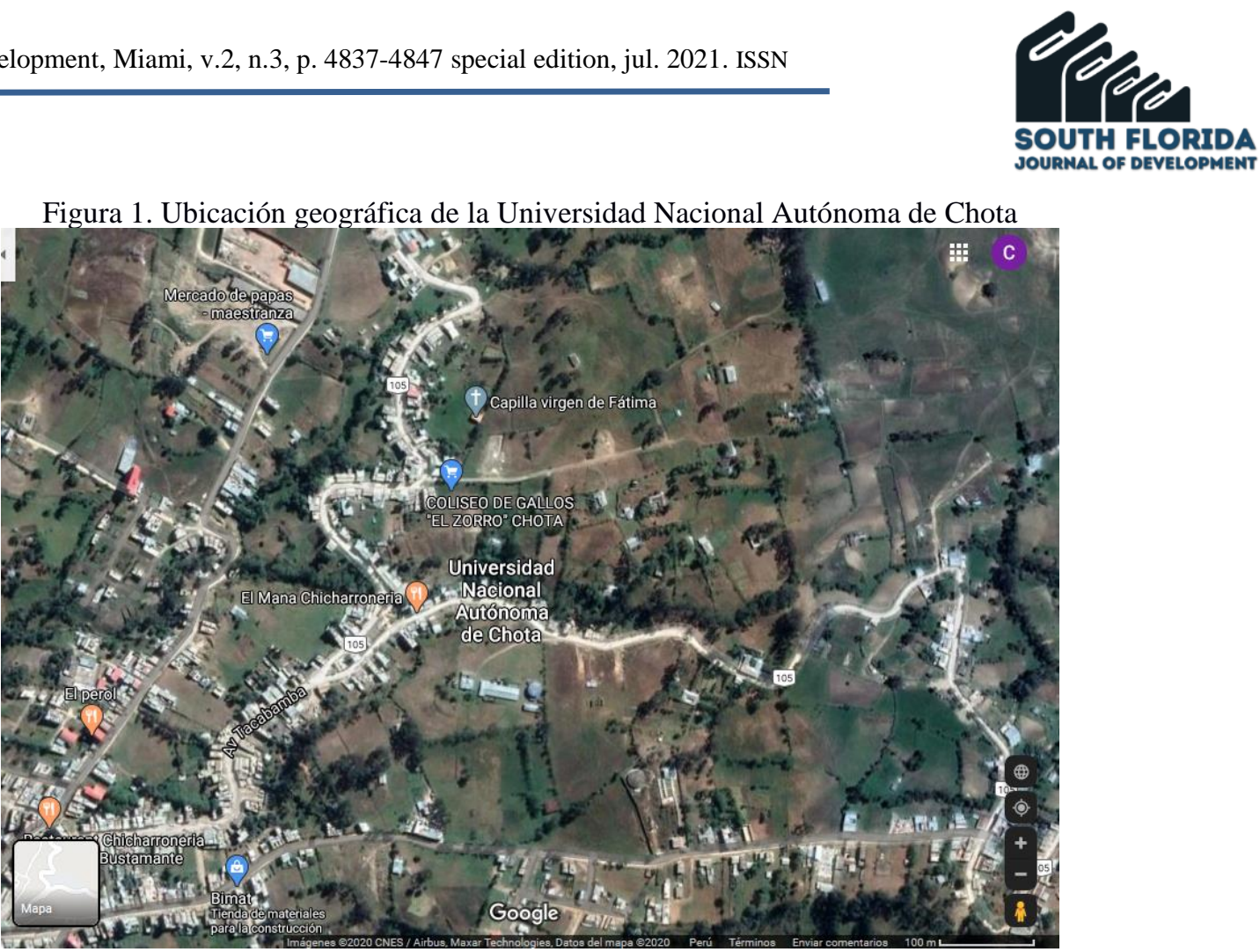

Fuente: Google maps (2020).

La Universidad Nacional de Jaén (UNJ), se convirtió en una realidad cuando el Congreso de la República aprueba la Ley 29304 el 12 de diciembre de 2008, creando la Universidad Nacional de Jaén, y publicándose 5 día después en la Ciudad de Jaén. Tres años después, el 22 de diciembre de 2011, la Resolución 647-2011-CONAFU autoriza el funcionamiento provisional de la UNJ con las cinco carreras profesionales que a la fecha existen: Ingeniería Civil, Ingeniería Mecánica y Eléctrica, Ingeniería de Industrias Alimentarias, Ingeniería Forestal y Ambiental y Tecnología Médica con especialidad en Laboratorio Clínico (UNJ, 2018).

Figura 2. Ubicación geográfica de la Universidad Nacional de Jaén

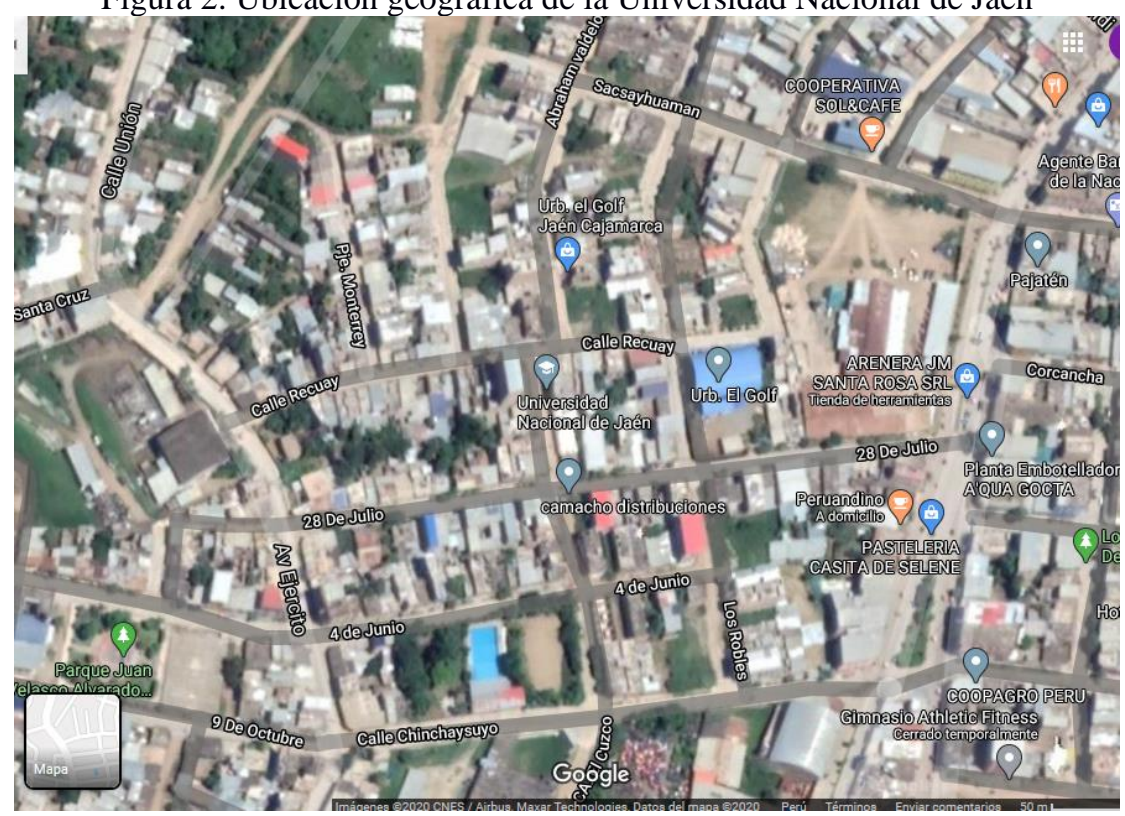

Fuente: Google maps (2020). 


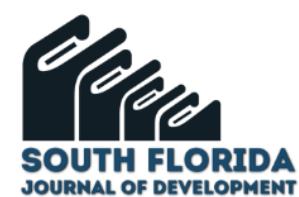

La Universidad Nacional de Cajamarca (UNC), fue aprobada el 13 de febrero del año 1962, cuando se promulga la Ley No. 14015. El 14 de julio del mismo año inicia su funcionamiento, con una planificación de seis escuelas: Medicina rural, medicina veterinaria, pedagogía, minería y metalurgia, economía y organización de empresas; en nuestros días cuenta con otra estructura normativa, fundamentándose en la formación académica, investigación y proyección social, con diez facultades: Educación, ingeniería, ciencias agrícolas y forestales, ciencias de la salud, ciencias económicas, contables y administrativas, ciencias sociales, ciencias veterinarias, zootencia, medicina humana, derecho y ciencias políticas..., la universidad cuenta actualmente con 4 subsedes en distintas provincias de la región: Jaén, Bambamarca, Celendín y Cajabamba (UNC, 2020).

Figura 3. Ubicación geográfica de la Universidad Nacional de Cajamarca

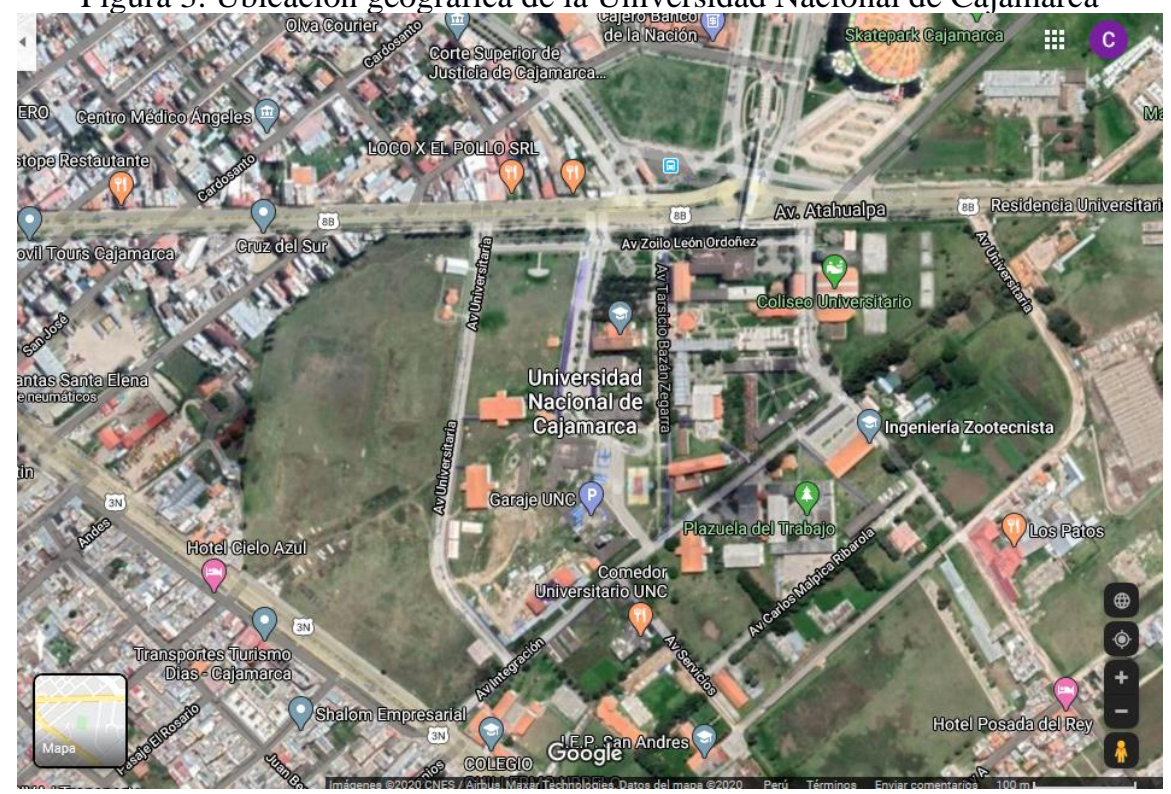

Fuente: Google maps (2020).

Tabla 1 Universidades nacionales de la Región Cajamarca.

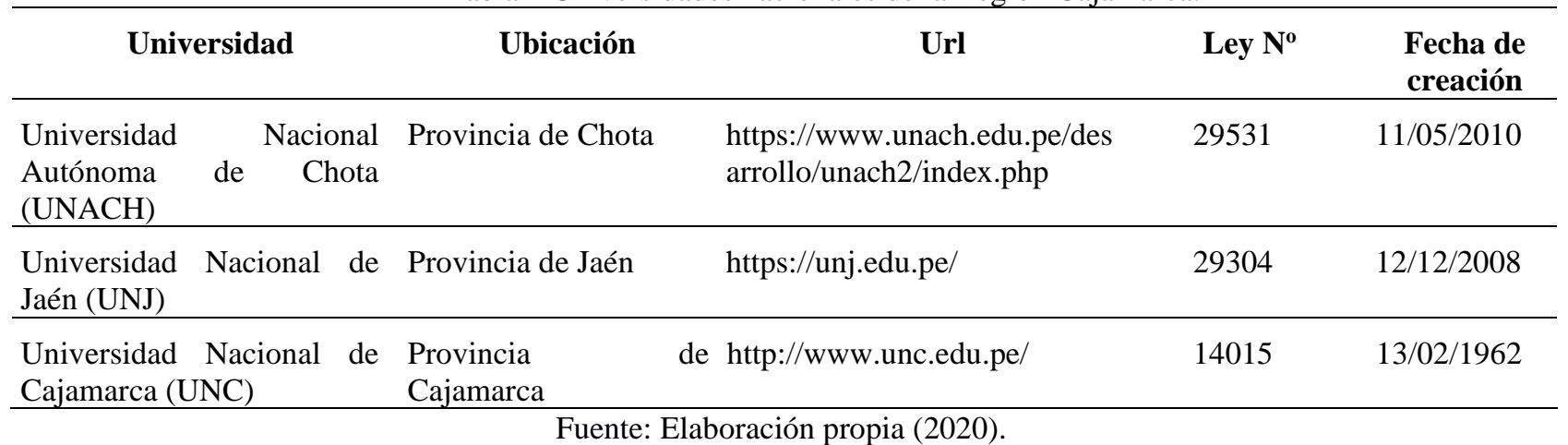


Tabla 2 Revistas científicas de las universidades nacionales de la Región Cajamarca.

\begin{tabular}{|c|c|c|c|c|c|}
\hline Universidad & Nombre & $\begin{array}{l}\text { Fecha del } \\
\text { primer } \\
\text { número } \\
\end{array}$ & Url & Frecuencia & Disciplinas \\
\hline $\begin{array}{l}\text { Universidad } \\
\text { Autónoma de } \\
\text { (UNACH) }\end{array}$ & $\begin{array}{c}\text { Revista } \\
\text { Ciencia } \\
\text { Nor@ndina }\end{array}$ & $\begin{array}{l}\text { Enero- } \\
2018\end{array}$ & $\begin{array}{l}\text { http://unach.edu. } \\
\text { pe/rcnorandina/i } \\
\text { ndex.php/ciencia } \\
\text { norandina }\end{array}$ & Semestral & $\begin{array}{l}\text { Ciencias de la salud. } \\
\text { Ciencia y } \\
\text { tecnología. } \\
\text { Recursos naturales y } \\
\text { medio ambiente. } \\
\text { Alimentos } \\
\text { biotecnología. } \\
\text { Ciencias contables y } \\
\text { administrativas }\end{array}$ \\
\hline $\begin{array}{l}\text { Universidad Nacional } \\
\text { de Jaén (UNJ) }\end{array}$ & $\begin{array}{c}\text { Revista } \\
\text { Científica } \\
\text { Pakamuros }\end{array}$ & $\begin{array}{l}\text { Enero - Junio, } \\
2013\end{array}$ & $\begin{array}{l}\text { http://revistas.unj. } \\
\text { edu.pe/index.php/ } \\
\text { pakamuros }\end{array}$ & Trimestral & $\begin{array}{l}\text { Ingeniería, } \\
\text { Educación y Salud }\end{array}$ \\
\hline $\begin{array}{l}\text { Universidad Nacional } \\
\text { de Cajamarca (UNC) }\end{array}$ & $\begin{array}{c}\text { Revista } \\
\text { Científica } \\
\text { Caxamarca }\end{array}$ & Mayo de 2018 & $\begin{array}{l}\text { http://revistas.unc. } \\
\text { edu.pe/ }\end{array}$ & Anual & $\begin{array}{l}\text { Ciencias } \\
\text { Veterinarias, } \\
\text { Ingeniería en } \\
\text { Ciencias Pecuarias } \\
\text {, Ciencias Agrarias } \\
\text { Ciencias de la } \\
\text { Salud }\end{array}$ \\
\hline
\end{tabular}

Fuente: Elaboración propia (2020).

\section{METODOLOGÍA}

Las revistas científicas de las tres universidades nacionales de la Región Cajamarca, fueron analizadas empleando la metodología de Latindex (características de calidad del Catálogo 2.0). La metodología de Latindex, considera un total de 38 características, agrupadas en básicas, presentación, gestión y política editorial, contenido y revistas en línea.

Las características de calidad del Catálogo 2.0 (Metodología), versión 3, 1 de junio de 2020, tiene el objetivo de esta nueva lista de características es reforzar el papel pedagógico y didáctico que Latindex ha venido desempeñando con las personas editoras desde la aparición del Catálogo en 2002. La actualización de la metodología resultaba necesaria para adaptarla a los nuevos procesos de comunicación científica que se están dando en el mundo a través de revistas disponibles en línea. Con esta nueva metodología -y con en el reacomodo de características, especialmente las que han sido integradas al grupo de "obligatorias"-, se busca que las personas editoras estén informadas sobre las reglas que privarán en la conformación del Catálogo 2.0 en cada uno de los centros nacionales (Latindex, 2020). 


\section{RESULTADOS}

A continuación, se revelan los resultados alcanzados, después de utilizar la metodología de Latindex. El análisis de las revistas científicas de las universidades nacionales de la Región Cajamarca, se realizó durante el mes de agosto de 2020.

Tabla 3 Catálogo 2.0 Listado de características

\begin{tabular}{|c|c|c|c|}
\hline CARACTERÍSTICAS BÁSICAS & UNACH & UNJ & UNC \\
\hline 1. Responsables editoriales & $\mathrm{X}$ & $\mathrm{X}$ & $\mathrm{X}$ \\
\hline 2. Generación continua de contenidos & $\mathrm{X}$ & $\mathrm{X}$ & $\mathrm{X}$ \\
\hline 3. Identificación de la autoría & $\mathrm{X}$ & $\mathrm{X}$ & $\mathrm{X}$ \\
\hline 4. Entidad editora de la revista & $\mathrm{X}$ & $\mathrm{X}$ & $\mathrm{X}$ \\
\hline 5. Instrucciones para publicar & $\overline{\mathrm{X}}$ & $\bar{X}$ & $\overline{\mathrm{X}}$ \\
\hline 6. Sistema de arbitraje & $\mathrm{X}$ & $\mathrm{X}$ & $\mathrm{X}$ \\
\hline 7. ISSN & $\mathrm{X}$ & $\mathrm{X}$ & $\mathrm{X}$ \\
\hline \multicolumn{4}{|l|}{ CARACTERÍSTICAS DE PRESENTACIÓN } \\
\hline 8. Navegación y funcionalidad en el acceso a contenidos & $\mathrm{X}$ & $\mathrm{X}$ & $\mathrm{X}$ \\
\hline 9. Acceso histórico al contenido & $\mathrm{X}$ & $\mathrm{X}$ & $\mathrm{X}$ \\
\hline 10. Mención de periodicidad & $\mathrm{X}$ & $\mathrm{X}$ & $\mathrm{X}$ \\
\hline 11. Membrete bibliográfico al inicio del artículo & $\mathrm{X}$ & $\mathrm{X}$ & $\mathrm{X}$ \\
\hline $\begin{array}{l}\text { 12. Afiliación institucional de los miembros de los cuerpos } \\
\text { editoriales }\end{array}$ & $\mathrm{X}$ & $\mathrm{X}$ & $\bar{X}$ \\
\hline 13. Afiliación de las personas autoras & $\mathrm{X}$ & $\mathrm{X}$ & $\mathrm{X}$ \\
\hline 14. Fechas de recepción y aceptación de originales & $\mathrm{X}$ & $\bar{X}$ & $\bar{X}$ \\
\hline
\end{tabular}

\section{CARACTERÍSTICAS DE GESTIÓN Y POLÍTICA} EDITORIAL

\begin{tabular}{|c|c|c|c|}
\hline 15. Definición de la revista & $\mathrm{X}$ & $\mathrm{X}$ & $\mathrm{X}$ \\
\hline 16. Documentos con autoría externa & & $\mathrm{X}$ & \\
\hline 17. Apertura editorial & $\mathrm{X}$ & $\mathrm{X}$ & $\mathrm{X}$ \\
\hline 18. Servicios de información & $\mathrm{X}$ & $\mathrm{X}$ & $\mathrm{X}$ \\
\hline 19. Cumplimiento de periodicidad & $\mathrm{X}$ & $\mathrm{X}$ & $\mathrm{X}$ \\
\hline 20. Políticas de acceso y reuso & & & $\mathrm{X}$ \\
\hline 21. Código de ética & $\mathrm{X}$ & & \\
\hline 22. Detección de plagio & & $\mathrm{X}$ & \\
\hline \multicolumn{4}{|l|}{$\begin{array}{l}\text { CARACTERÍSTICAS DE CONTENIDO } \\
\end{array}$} \\
\hline 23. Contenido original & $\mathrm{X}$ & $\mathrm{X}$ & $\mathrm{X}$ \\
\hline 24. Referencias bibliográficas adoptando una norma & $\bar{X}$ & $\bar{X}$ & $\mathrm{X}$ \\
\hline 25. Exigencia de originalidad & $\overline{\mathrm{X}}$ & $\overline{\mathrm{X}}$ & $\overline{\mathrm{X}}$ \\
\hline 26. Resumen & $\overline{\mathrm{X}}$ & $\overline{\mathrm{X}}$ & $\mathrm{X}$ \\
\hline 27. Resumen en dos idiomas & $\mathrm{X}$ & $\mathrm{X}$ & $\mathrm{X}$ \\
\hline 28. Palabras clave & $\bar{X}$ & $\bar{X}$ & $\bar{X}$ \\
\hline 29. Palabras clave en dos idiomas & $\mathrm{X}$ & $\mathrm{X}$ & $\mathrm{X}$ \\
\hline 30. Cantidad de artículos publicados por año & $\mathrm{X}$ & $\mathrm{X}$ & $\bar{X}$ \\
\hline
\end{tabular}




\begin{tabular}{|c|c|c|c|}
\hline CARACTERÍSTICAS DE REVISTAS EN LÍNEA & & & \\
\hline 31. Uso de protocolos de interoperabilidad & $\mathrm{X}$ & $\mathrm{X}$ & $\mathrm{X}$ \\
\hline 32. Uso de diferentes formatos de edición & $\mathrm{X}$ & $\mathrm{X}$ & $\mathrm{X}$ \\
\hline 33. Servicios de valor agregado & $\mathrm{X}$ & $\mathrm{X}$ & $\mathrm{X}$ \\
\hline 34. Servicios de interactividad con la persona lectora & $\overline{\mathrm{X}}$ & $\overline{\mathrm{X}}$ & $\overline{\mathrm{X}}$ \\
\hline 35. Buscadores & $\mathrm{X}$ & $\mathrm{X}$ & $\mathrm{X}$ \\
\hline 36. Uso de identificadores uniformes de recursos & $\mathrm{X}$ & $\mathrm{X}$ & \\
\hline \multicolumn{4}{|l|}{ 37. Uso de estadísticas } \\
\hline 38. Políticas de preservación digital & & & \\
\hline
\end{tabular}

La revista científica de la Universidad Nacional Autónoma de Chota (UNACH) ha cumplido 33 características de la metodología de Latindex.

La revista científica de la Universidad Nacional de Jaén (UNJ) ha cumplido 34 características de la metodología de Latindex.

La revista científica de la Universidad Nacional de Cajamarca (UNC) ha cumplido 32 características de la metodología de Latindex.

Las revistas científicas de la UNC y UNJ han logrado su indexación en Latindex, mientras que de la UNACH no tiene indexación alguna, por lo que su visibilidad es mínima.

Para referirse a los resultados presentados en esta investigación, se tiene que indicar, que las tres revistas científicas cumplen con más de 30 características de la metodología de Latindex, lo cual demuestra el esfuerzo de los equipos editoriales por tener revistas científicas de calidad.

\section{CONCLUSIONES}

Con respecto al primer objetivo, se determinó que las revistas científicas de la Universidad Nacional de Cajamarca (UNC) y Universidad Nacional de Jaén (UNJ) han logrado su indexación en Latindex, mientras que de la Universidad Nacional Autónoma de Chota (UNACH) no tiene indexación alguna, por lo que su visibilidad es mínima.

Con respecto al segundo objetivo, se recomienda minimizar los índices de endogamia editorial y autoría de las revistas científicas de las universidades nacionales de la Región Cajamarca. Además, se debe indicar las políticas de acceso y reuso, detección de plagio, uso de estadísticas y políticas de preservación digital. 


\section{REFERENCIAS BIBLIOGRÁFICAS}

Aleixandre-Benavent, R., Ferrer A. y Peset, F. Compartir los recursos útiles para la investigación: datos abiertos (open data). Educación Médica. https://doi.org/10.1016/j.edumed.2019.07.004.

Barbón et al., O. (2019). La elaboración de la sección «Discusión» de artículos científicos originales. ¿Un reto para los docentes universitarios?. Educación Médica, Volume 20, Issue 6, pages 380-386. https://doi.org/10.1016/j.edumed.2017.12.011.

Carvalho, M., Lima, L. y Coeli, C. (2020). Ciencia en tiempos de pandemia. Cadernos de Saúde Pública, 36(4), e00055520. https://dx.doi.org/10.1590/0102-311x00055520

Casimiro, W., Casimiro, C. y Casimiro, J. Los posgrados y la investigación científica en las universidades peruanas. http://mendive.upr.edu.cu/index.php/MendiveUPR/article/view/1876

Centeno-Leguía, C., Morales-Concha, L., Lopez, C. \& Mejia, C. (2020). Mujeres científicas: características y factores asociados a la primera autoría y corresponsalía en revistas peruanas indizadas a SciELO, 2010-2015. Educación Médica, Volume 21, Issue 1, pages 17-23. https://doi.org/10.1016/j.edumed.2018.04.010.

Cruz, F. et al. (2020). Publicación de tesis sustentadas en un pregrado de ciencias de la salud de una universidad colombiana, 2012-2017. Prevalencia y factores relacionados. Educación Médica. https://doi.org/10.1016/j.edumed.2019.11.006.

Chachaima-Mar, J., Fernández-Guzmán, D. y Atamari-Anahui, N. (2019). Publicación científica de docentes de una escuela de medicina peruana: frecuencia y características asociadas. Educación Médica, Volume 20, Supplement 2, pages 2-9. https://doi.org/10.1016/j.edumed.2017.10.024.

Gonzalez-Argote, J. y Garcia-Rivero, A. (2020). Repositorio de investigaciones estudiantiles: tarea necesaria y transcendental. Educación Médica, Volume 21, Issue 3, pages 212-217. https://doi.org/10.1016/j.edumed.2018.04.014.

Gregorio-Chaviano, O. (2018). Evaluación y clasificación de revistas científicas: reflexiones en torno a retos y perspectivas para Latinoamérica. Revista Lasallista de Investigación, vol. 15, núm. 1. https://doi.org/10.22507/rli.v15n1a12

Hernández, I. y Navia, A. (2019). La importancia del impulso a la investigación científica en la Sudamérica emergente. Cirugía Plástica Ibero-Latinoamericana, 45(2), 207-208. https://dx.doi.org/10.4321/s0376-78922019000200015

Latindex (2020). Documentos de Latindex. https://www.latindex.org/latindex/meto2

Miguel, S. (2011). Revistas y producción científica de América Latina y el Caribe: su visibilidad en SciELO, RedALyC y SCOPUS. Revista Interamericana de Bibliotecología, 2011, vol. 34, no. 2, p. 187199.

Rodríguez, R., Socorro, A., y León, J. (2019). Estrategias para la indización de revistas académicas en Ciencias Aplicadas. Revista Universidad y Sociedad, 11(5), 81-88. http://scielo.sld.cu/scielo.php?script=sci_arttext\&pid=S2218-36202019000500081\&lng=es\&tlng=es. 
UNACH (2018). Reseña histórica, Universidad Nacional Autónoma de Chota. https://www.unach.edu.pe/desarrollo/unach2/index.php/nosotros/historia

UNC (2020). Reseña Histórica UNC. http://www.unc.edu.pe/Institucional/ResenaHistorica

UNESCO (2020). La ciencia es y será de la gente. https://es.unesco.org/news/ciencia-es-y-sera-gente UNJ (2018). Reseña Histórica de la Universidad Nacional de Jaén. https://www.unj.edu.pe/index.php/universidad-nacional-jaen/organizacion/historia-universidadnacional-jaen

Venegas, V., Esquivel, J., y Turpo-Gebera, O. (2019). Reflexiones sobre la investigación educativa y la investigación formativa en la Universidad Peruana. Conrado, 15(70), 444-454. Recuperado de http://scielo.sld.cu/scielo.php?script=sci_arttext\&pid=S1990-86442019000500444\&lng=es\&tlng=es. 